\title{
Effector Form Design for 1DOF Planar Actuation
}

\author{
Alberto Rodriguez and Matthew T. Mason \\ The Robotics Institute - Carnegie Mellon University \\ albertorecmu. edu, matt.masonecs. cmu. edu
}

\begin{abstract}
Given a desired function for an effector, what is its appropriate shape? This paper formulates mechanical function as a product of both effector's shape and motion, and, assuming a fixed motion model, explores the role of shape in satisfying it. We assume that the desired mechanical function is expressed as a set of constraints on the geometry of contact, and develop the tools for transforming these constraints into an effector shape. A previous paper [1] addressed the special case of revolute or prismatic fingers. This paper develops the more general case, including all smooth 1DOF planar mechanisms. The technique is illustrated with the design of finger shapes to improve the stability of a planar grasp of an object.
\end{abstract}

\section{INTRODUCTION}

The connection between function and effector shape is ubiquitous in manipulation and all of robotics (Figure 1). It plays an important role in determining the reaction of an object to contact and has the potential to express mechanical intelligence, yet the design of effector shape is neglected relative to other areas of manipulation research.

This paper explores design of effector shape. We assume that the desired function can be described by a set of constraints on contact geometry, and develop the techniques for integrating those constraints to produce an effector shape. We illustrate the approach by an example: deriving a finger shape that improves the stability of a grasp.

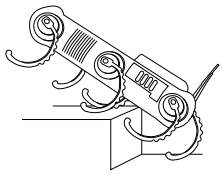

(a)

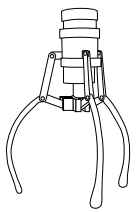

(e)

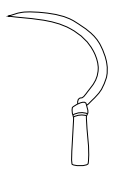

(b)

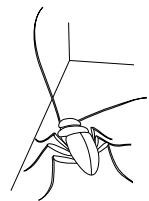

(f)

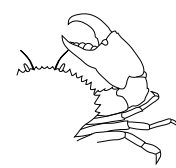

(c)

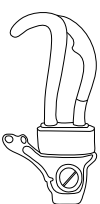

(g)

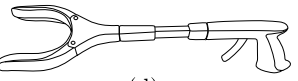

(d)

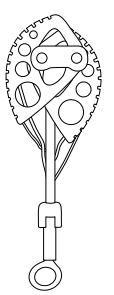

(i)
Fig. 1. Examples of effectors whose shape might have a relevant role in mechanical function: (a) hexapod robot "RHex", (b) sickle, (c) crab pincer, (d) manual gripper "Grip'n Grab", (e) claw crane, (f) cockroach antennae, (g) prosthetic hook, (h) prosthetic leg, and (i) rock-climbing cam.

This work was supported by National Science Foundation [NSF-IIS0916557] and Army Research Laboratory [W911NF-10-2-0016]. This work does not necessarily reflect the position or the policy of the U.S. Government or ARL. No official endorsement should be inferred.
The basic idea of using contact constraints to express function is illustrated in Figure 2, with the example task of moving a disk along a given path. In an idealized frictionless quasistatic world, the effector should contact the disk with a contact normal along the path tangent as in Figure 2b. In the presence of friction or perturbations, the disk would surely deviate from the desired path. In that case, the "corrective" contact constraints shown in Figure $2 \mathrm{c}$ and are chosen to stabilize its motion. Examining these additional corrective contact constraints would allow one to reject a point pusher, or a flat pusher, and instead choose a cupped shape. Reducing a desired function to contact constraints is quite common. For example we often use it to explain the stability of an object at rest on a table.

(a)

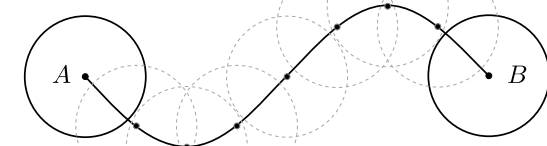

(b)

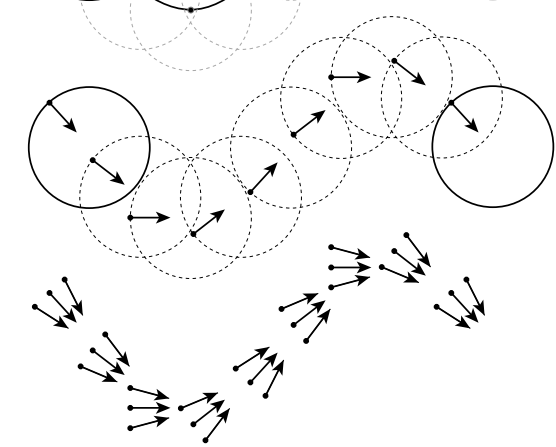

Fig. 2. An example task represented by a set of contact constraints. (a) The goal is to move the disk from A to B along the given path. (b) For the disk to follow the path, the effector must push it along the path tangent, which gives a continuum of contact constraints. (c) Extra "corrective" constraints to make the push robust to perturbations, such as friction.

In previous work [1] we explored the design of effectors yielding contact geometry invariant with respect to the scale or the pose of an object. An example is given in Figure 3, a pickup tool designed so that the grasp geometry is invariant as the disk ascends to the palm. Our techniques applied only to the special case of effectors actuated by revolute or prismatic joints. This paper develops a more general formulation that covers arbitrary smooth 1DOF mechanisms. A look at [1], in particular Figure 6, might be of help to understand the general idea. We also explore design goals 

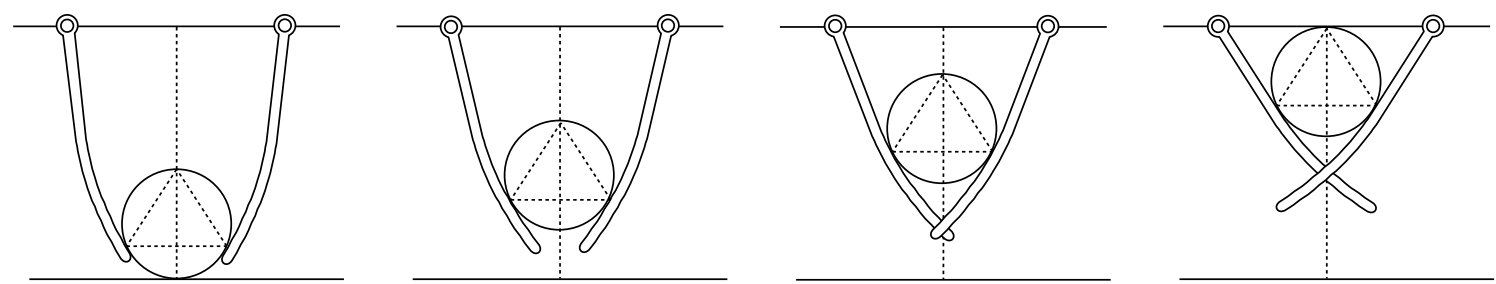

Fig. 3. A planar pickup tool, designed to give invariant grasp geometry as the disk's pose varies along the vertical. [1]

other than grasp invariance, such as improving the stability of a grasp.

In general, we will express a design goal as a set of contact constraints. The central problem addressed in this paper is to design a shape so that each constraint is satisfied for some configuration of the mechanism; the main challenge lies in not knowing a priori what configuration of the effector should satisfy each constraint; and the key insight will be to employ an extended space, the cartesian product of the workspace and the configuration space of the mechanism.

The paper's organization is:

1) introduction (done),

2) related work,

3) formal models of effector shape and motion,

4) formal models of geometric contact constraints and related operations on constraints,

5) formulation of the Shape for Contact problem,

6) example application to an effector design problem,

7) further discussion on shape and contact.

\section{RELATED WORK}

There is a long history of using mechanical design in place of online computation, especially in that period before computers were available. The principle of replacing computation with mechanical design has been called "mechanical intelligence" (Ulrich [2]), "hard automation" (Canny and Goldberg [3]) "morphological computation" (Pfeifer and Iida [4]) or "adaptive mechanics" (Gosselin [5]).

Mechanisms such as cams, linkages or gears have been used for centuries to transform actuation into carefully planned motion. One notable application is the design of kinematic and passive-dynamic mechanisms to produce walking machines (Raibert [6], McGeer [7], Collins et al. [8], Gomes and Ruina [9]), which dates back at least to the 1800 's with a walking device based on Chebyshev's linkage to transform rotational motion into approximate straight-line motion (Lucas [10]). In an example particularly pertinent to the present work McGeer [7] examines foot shape and the resulting evolution of contact between foot and ground.

Perhaps most relevant to this paper is the desire to build simple yet capable robotic hands (Hirose and Umetani [11], Ulrich [2], Dollar and Howe [12], Birglen et al. [13], Rodriguez et al. [14], Mason et al. [15]). Compliance and underactuation take care of shape adaptation which in turn reduces the need for complex mechanics and controls.

In this paper, rather than motion or compliance, we target the shape of a mechanism as possible conveyor of function.
In general, shape alone is not enough to solve a manipulation problem, however, it is a "cheap" design freedom with potential benefits both in terms of simplicity and robustness. The circular leg design by Moore et al. [16] for the robotic hexapod Rhex (Saranli et al. [17]) is a clear example of shape serving simplicity. Robustness is also a potential benefit of shape design, for example in the design of rock climbingcams (Jardine [18]) designed to provide sufficient grip over a wide range of crack widths (Rodriguez and Mason [1]).

In the context of grasping and the design of robotic hands, shape has rarely played an important role. The most common approach is to rely on grasp planners to choose fixed contact points based on precise knowledge of object shape and pose. Even when sliding or rolling contacts are modeled, they are seldom exploited to design functional phalanx shape.

Dollar and Howe [19] reviewed the designs of 20 different compliant and underactuated robotic hands, all employing either cylindrical or flat straight fingers and no cited principle guiding the designs of their shapes. Theobald et al. [20] is one of the few exceptions with the design of a gripper for autonomous rock acquisition with curved fingers. More recently, similar to the application explored in this paper, Kragten et al. [21] considered curving the contact area of distal phalanges to improve the stability of precision grasps.

Shape synthesis has also been studied in the context of automation, especially in the context of part feeding and automated assembly. Traps, fences and chamfers are examples of features where mechanical interaction and response to contact can be planned in advance and hardcoded in the mechanism itself. Boothroyd and Dewhurst [22] presented a comprehensive collection of mechanical feeding and orienting techniques. Berretty et al. [23] analyzed the interaction between objects and traps to automate the design of vibratory bowl feeders. With a similar goal Peshkin and Sanderson [24] and Wiegley et al. [25] worked on the design of fences to reorient parts. Brokowski et al. [26] proposed adding curved tails to the end of a fence to reduce the object's pose uncertainty. Still in the context of part feeding, Zhang and Goldberg [27] systematized the design of the blades of a parallel jaw gripper to passively align parts in the vertical plane. Whitney et al. [28] designed curved chamfers to simplify the assembly of rigid parts.

Reuleaux [29] introduced the concept of kinematic pair, as an attempt to abstract motion constraints between contacting bodies. For ideal joints such as prismatic or revolute (lower pairs), shape is of little consequence. But for pairs where contact is maintained between curved surfaces like 
in the case of cams or gears (higher pairs), shape plays a key role. Several works in the early 90s approached the problem of qualitative shape understanding for kinematic pairs (Joskowicz [30], Joskowicz and Addanki [31], Faltings [32], Forbus et al. [33]), with the goal of understanding the effect in the configuration space of small alterations to the shape of the contacting bodies. Gupta and Jakiela [34] designed kinematic pairs by sweeping a fixed shape along a predefined path and numerically "carving" the other shape. Inspired by applications such as vibratory bowl feeders and part mating, Caine $[35,36]$ studied the design of shape from motion constraints.

In contrast, this paper represents mechanical function as a collection of geometric contact constraints to be satisfied by the shape of the effector. Contact kinematics, the study of how contact location changes with object motion, is essential to understand how contact constraints and mechanical function are related. Cai and Roth [37] studied the motion of the contact between two objects that roll-slide on each other and Montana [38] provided a more formal approach to the same problem.

\section{END EFFECTORS: ShAPE AND Motion}

The kinematic function of an end effector is determined, in great part, by its motion and its shape. As illustrated in Figure 4, both shape and motion have an impact in that kinematic function. In this section we formalize the model of an effector, and introduce the concepts of motion field, motion orbit, and orbit space.
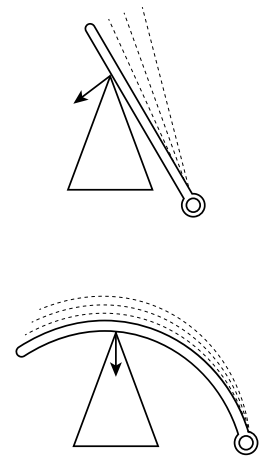

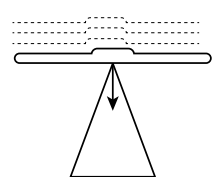

(a)

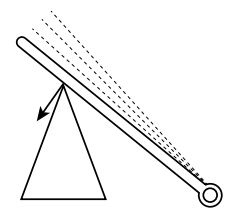

(b)
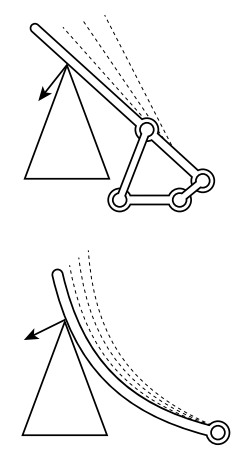

Fig. 4. (a) Three actuation mechanisms and (b) three effector shapes contacting an object at a given point. The expected reaction of the object to that contact varies both with the motion and the shape of the effector.

The respective contributions of shape and motion to the function of an effector are intertwined. The suitability of a shape depends on motion, and vice versa. In this paper we assume a fixed given effector motion and address the shape synthesis problem.

We make three simplifying assumptions on the effector:

- The effector is rigid. There is no compliance on the shape of the effector nor on the actuation mechanism.

- The effector is planar. Both the shape and the actuation mechanism lie on a planar workspace $\mathcal{W} \simeq \mathbb{R}^{2}$.
- The effector actuation is via a $1 \mathrm{DOF}$ smooth mechanism.

We make no assumptions on the object, other that it is possible to describe the desired task as a set of contacts.

We formally define now the concept of effector. Let $\mathcal{W}$ be a planar workspace, let $s \in S=\left[s_{\min }, s_{\max }\right]$ be the shape parameter, parametrizing the effector shape, and let $t \in$ $T=\left[t_{\min }, t_{\mathrm{max}}\right]$ be the motion parameter, parametrizing the configuration space of the mechanism driving the effector. Without loss of generality, we assume that $0 \in T$.

Definition 1 (Effector): An effector $E$ is a smooth map from shape and motion parameters to workspace points $E$ : $S \times T \mapsto \mathcal{W}$, where for a fixed $t, E(\cdot, t)$ parametrizes with unit speed a rigid transformation of the curve $E(s, 0)$.

$E$ describes the motion (parametrized by $t$ ) of a rigid curve (parametrized by $s$ ) as actuated by the mechanism, so that $E\left(s, t_{0}\right)$ is the effector shape at configuration $t_{0}$. For simplicity, we will refer by shape to the curve $E(s, 0)$.

\section{A. Motion Field}

The motion field is a representation of the motion imposed by a mechanism. For each point $p$ on the effector shape in pose $t_{0}$, we define the velocity $v_{p}$ by differentiating $E(s, t)$ with respect to $t$ and holding the shape parameter $s$ fixed. Note that $v_{p}$ is independent of the shape of the effector (we differentiate with respect to $t$ ), and can be defined for any $p \in \mathcal{W}$ and any $t \in T$ simply by considering an effector that crosses $p$ at configuration $t$.

For reasons that will be apparent, we consider an extended space, the cartesian product of the workspace and the mechanism configuration space $\mathcal{W} \times T$. We define then:

Definition 2 (Motion Field): Motion field $\mathcal{M}$ is the vector field representing the direction of imposed effector motion:

$$
\begin{aligned}
\mathcal{M}: \mathcal{W} \times T & \rightarrow \mathcal{T}(\mathcal{W} \times T) \\
(p, t) & \mapsto\left(v_{p}, 1\right)
\end{aligned}
$$

where $\mathcal{T}(\mathcal{W} \times T)$ is the tangent bundle of $\mathcal{W} \times T$.

Note that the last component of $\mathcal{M}(p, t)$ is always 1 . This reflects the fact that the effector is continuously actuated by the mechanism. Figure 5 shows the motion fields of three different actuation mechanisms: a rotational joint, a Hoekens linkage, and an elliptic trammel.

In the following subsections we see that the motion field $\mathcal{M}$ partitions the space $\mathcal{W} \times T$ into disjoint motion orbits. Those motion orbits will constitute the domain of influence of contact constraints.

\section{B. Motion Orbits}

In differential geometry, the flow $\Phi$ of a smooth vector field $V$ on a manifold $\mathcal{N}$ is defined, for every point $q \in \mathcal{N}$, as the trajectory that a particle at $q$ would describe following an integral curve of $V$. Let $\Phi_{\mathcal{M}}$ be the flow of the motion field $\mathcal{M}$ :

$$
\begin{aligned}
\Phi_{\mathcal{M}}:(\mathcal{W} \times T) \times \mathbb{R} & \rightarrow \mathcal{W} \times T \\
((p, t), u) & \mapsto \Phi_{\mathcal{M}}((p, t), u)
\end{aligned}
$$

where $\Phi_{\mathcal{M}}((p, t), \cdot)$ is the unique integral curve of $\mathcal{M}$ passing through $(p, t)$. Intuitively, the motion flow $\Phi_{\mathcal{M}}$ describes 


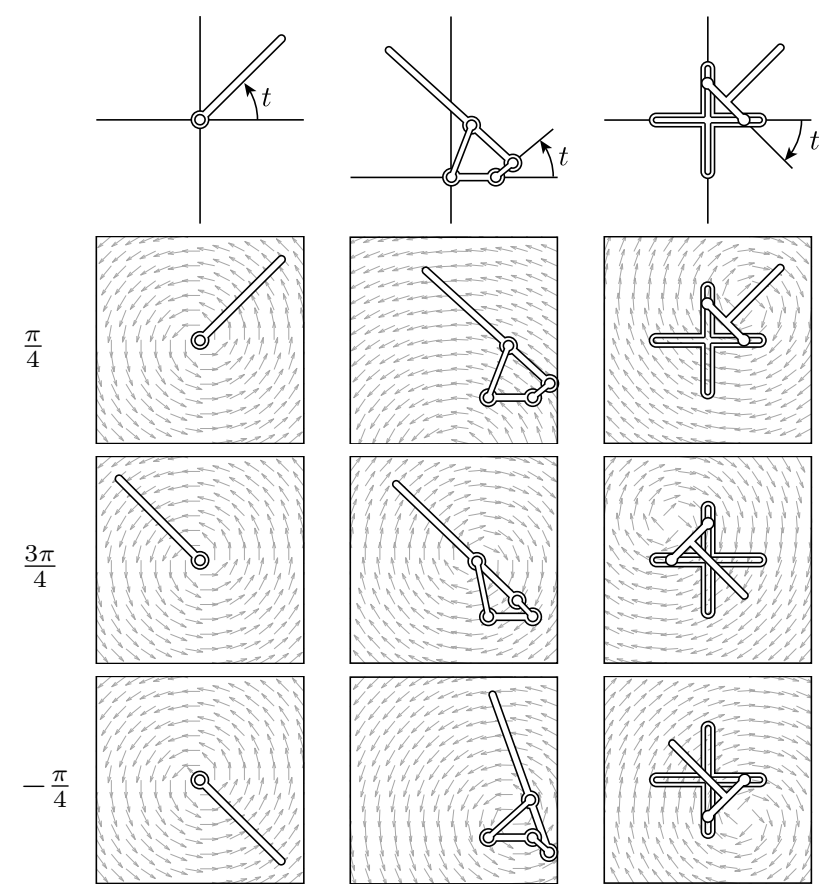

Fig. 5. Motion field for three mechanisms: (left) rotational joint, (middle) Hoekens linkage, and (right) elliptic trammel. Rows are slices $t=\frac{\pi}{4}$, $t=\frac{3 \pi}{4}$, and $t=-\frac{\pi}{4}$ of the motion field. Note that slices $t=$ const are the motion field of a rotational joint at the instantaneous center of rotation. Note also that the motion field of a revolute joint is invariant with $t$.

the trajectory in $\mathcal{W} \times T$ followed by an effector particle positioned at $p$ when the mechanism starts at configuration $t$. We define the associated motion orbit as:

Definition 3 (Motion Orbit): The motion orbit of a point $(p, t) \in \mathcal{W} \times T$, under the motion flow $\Phi_{\mathcal{M}}$, is the set $\Phi_{(p, t)}=\left\{\Phi_{\mathcal{M}}((p, t), u): u \in \mathbb{R}\right\}$.

Note that, for the case of $1 \mathrm{DOF}$ effectors, the projections of the motion orbits from $\mathcal{W} \times T$ to the workspace $\mathcal{W}$ are also known as the coupler curves of the mechanism. Figure 6 shows that projection for three initial values of the motion parameter $t$ and three different mechanisms: a rotational joint, a Hoekens linkage, and an elliptic trammel.

\section{Space of Orbits}

In this section we study the structure of the set of motion orbits, which will later be used in Section IV to formalize the concept of contact constraint. Recall now that motion orbits are the integral curves of the motion field $\mathcal{M}$, and that they are defined for all $(p, t) \in \mathcal{W} \times T$.

It is always the case for a smooth non-vanishing vector field that its integral curves define a 1-dimensional foliation of the space. Intuitively, a foliation is a decomposition of the space into "parallel" subspaces of smaller dimension, like decomposing a plane into parallel lines, or 3D space into parallel planes. In particular, a 1-dimensional foliation is a decomposition of the space into non-intersecting curves.

In our case, $\mathcal{M}$ is a never-vanishing smooth vector field, since the third component is constant equal to 1 . Hence, the set of motion orbits decomposes the space $\mathcal{W} \times T$ into the union of non-intersecting curves. This allows us to define an

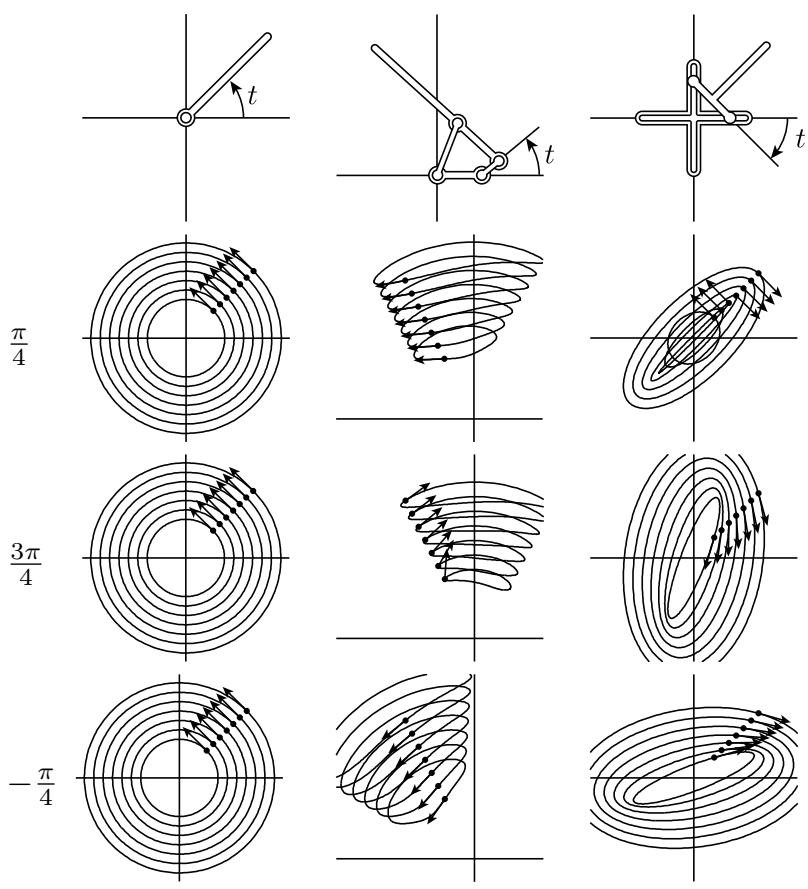

Fig. 6. Motion orbits for three mechanisms: (left) rotational joint, (middle) Hoekens linkage, and (right) elliptic trammel. The figure shows the projection of the motion orbits to the workspace $\mathcal{W}$ for three different initial mechanism configurations: $t=\frac{\pi}{4}, t=\frac{3 \pi}{4}$ and $t=-\frac{\pi}{4}$. Note that the motion orbits of a rotational joint are invariant with $t$.

equivalence relationship $\sim$, where two points in $\mathcal{W} \times T$ are equivalent, iff they share the same motion orbit:

$$
\left(p, t_{1}\right) \sim\left(q, t_{2}\right) \quad \Longleftrightarrow \quad \Phi_{\left(p, t_{1}\right)}=\Phi_{\left(q, t_{2}\right)}
$$

The space of orbits is then defined as:

Definition 4 (Orbit Space): The orbit space is the quotient space $\mathcal{O}=(\mathcal{W} \times T) / \sim$ where each element in $\mathcal{O}$ is representative of all points equivalent to each other.

For the purpose of visualization, we chose a single point from each class to represent it. If chosen properly, those representative points can form a lower dimensional subspace or section in $\mathcal{W} \times T$ easy to visualize.

Sections that are transversal to the motion flow $\Phi_{\mathcal{M}}$ and are crossed once and only once by each motion orbit are specially appropriate. An example is the set $\left\{t=t_{0}\right\}$ through which all orbits are guaranteed to cross once and only once, given that they are strictly monotonic in $t$. The section $\{t=0\}$ is a natural choice, given that in Section III we refer by effector shape to the curve $E(\cdot, 0)$, using $t=0$ as the reference mechanism configuration. Note also that the section $\{t=0\}$, i.e. the set $\mathcal{W} \times\{0\}$, is pointwise equivalent to $\mathcal{W}$. Hence we can think of the effector shape as a curve both in the workspace $\mathcal{W}$ or in the orbit space $\mathcal{O}$.

The characterization of shape and motion in this section leads us in Section IV to the argument that the domain of influence of contact constraints are entire motion orbits. This will allow us to transport contact constraints to a single mechanism configuration (the orbit space), and overcome the main challenge of not knowing a priori what configuration of the effector should satisfy what constraint. 


\section{Contact Constraints}

In this section we formalize contact constraints, and define important operations on them. For simplicity of exposition, in this paper we only refer to first order contact constraints. That is, we will only care about matching the tangents of object and effector. However, in a very similar fashion we can also impose higher order constraints, such as the curvature of the effector. Also note that, if required, we can always approximate higher order constraints by a combination of first order ones, as in Figure 2c.

We begin with the definition of two types of constraints. Given a point $p$ in the workspace and a desired tangent $\omega$ :

Definition 5 (Contact Constraint): A contact constraint $(p, \omega) \in \mathcal{W} \times \mathbb{S O}(1)$ is satisfied by an effector $E(s, t)$ if there are $t_{0} \in T$ and $s_{0} \in S$ such that:

$$
E\left(s_{0}, t_{0}\right)=p \text { and }\left.\frac{\partial E\left(s, t_{0}\right)}{\partial s}\right|_{s=s_{0}}=\omega .
$$

Definition 6 (Shape Constraint): A shape constraint $\left(\left(p, t_{0}\right), \omega\right) \in(\mathcal{W} \times T) \times \mathbb{S O}(1)$ is satisfied by an effector $E(s, t)$ if at configuration $t_{0}$ there is $s_{0} \in S$ such that:

$$
E\left(s_{0}, t_{0}\right)=p \text { and }\left.\frac{\partial E\left(s, t_{0}\right)}{\partial s}\right|_{s=s_{0}}=\omega .
$$

A contact constraint is satisfied if there is any configuration of the effector that complies with the desired tangent. A shape constraint specifies a particular configuration of the effector. Thus there is a very simple relation between a contact constraint and a shape constraint. By definition, a contact constraint $(p, \omega)$ is satisfied if and only if at least one of the shape constraints in the set $\{((p, t), \omega): t \in T\}$ is satisfied.

\section{A. Constraint Propagation}

As mentioned in the introduction, the main challenge in enforcing contact constraints is not knowing for what configuration of the effector each constraint should be satisfied. However, it is easier to enforce shape constraints, since they specify a specific effector configuration. The key insight is to look at a contact constraint as the whole set of shape constraints it represents. Then to enforce $(p, \omega)$ we:

1) "transform" the set $\{((p, t), \omega): t \in T\}$ of shape constraints so they all apply to the same effector configuration, $t=0$; and

2) make sure one of them is satisfied.

To formalize the idea of transforming constraints, we first have to derive an expression for equivalent constraints. We will use the terms moving frame and fixed frame to refer to coordinate frames rigidly attached respectively to the effector and to the workspace, as in Figure 7a.

Consider two equivalent points $\left(p_{1}, t_{1}\right) \sim\left(p_{2}, t_{2}\right)$ in the extended space $\mathcal{W} \times T$ of an effector. Equivalent points share the same orbit, and therefore the same effector particle that crosses $p_{1}$ at configuration $t_{1}$, also crosses $p_{2}$ at $t_{2}$. The tangents $\omega_{1}$ and $\omega_{2}$ of the effector at those two points are related, they are identical in the moving frame. We will say that the constraints $\left(\left(p_{1}, t_{1}\right), \omega_{1}\right)$ and $\left(\left(p_{2}, t_{2}\right), \omega_{2}\right)$ are equivalent: an effector satisfies one iff it satisfies the other.
By constraint propagation we mean the process that transforms constraint $\left(\left(p_{1}, t_{1}\right), \omega_{1}\right)$ into the equivalent constraint $\left(\left(p_{2}, t_{2}\right), \omega_{2}\right)$, which, by construction, is defined between any pair of equivalent points, Figure $7 \mathrm{~b}$. We note by $P_{\left(p_{1}, t_{1}\right) \rightarrow\left(p_{2}, t_{2}\right)}(\cdot)$ the function that maps tangent $\omega_{1}$ to $\omega_{2}$. In general we will propagate shape constraints to $t=0$.
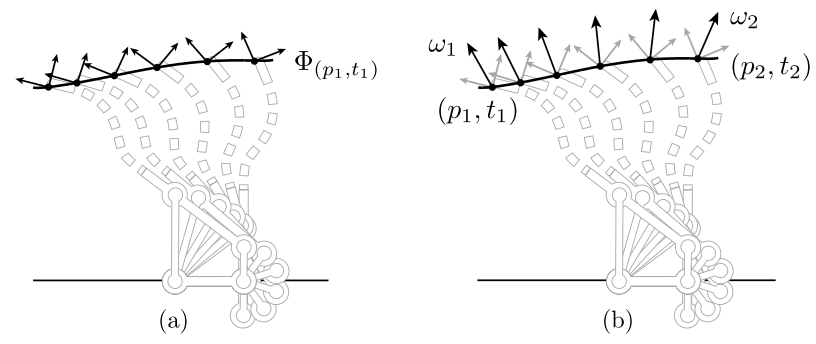

Fig. 7. (a) Moving frame for a Hoekens linkage between configurations $t_{1}=\frac{\pi}{2}$ and $t_{2}=-\frac{\pi}{2}$. The moving frame changes as if rigidly attached to the mechanism. (b) The propagation of the tangential constraint $\omega_{1}$ at point $\left(p_{1}, t_{1}\right)$ to $\omega_{2}$ at point $\left(p_{2}, t_{2}\right)$ is so that the constraint is held invariant in the moving frame $\left(\omega_{2}=P_{\left(p_{1}, t_{1}\right) \rightarrow\left(p_{2}, t_{2}\right)}\left(\omega_{1}\right)\right)$.

An important consequence of constraint propagation is that, by construction, an effector satisfies a shape constraint $((p, t), \omega)$ if and only if it satisfies any of the shape constraints propagated within the same orbit:

$$
((p, t), \omega) \Leftrightarrow\left\{\left(\left(q, t^{\prime}\right), P_{(p, t) \rightarrow\left(q, t^{\prime}\right)}(\omega)\right)\right\}_{\left(q, t^{\prime}\right) \in \Phi_{(p, t)}}
$$

Then, we say the domain of influence of a shape constraint is the entire motion orbit it represents. We can only impose one constraint per motion orbit.

\section{B. Constraint Locus}

In this section we use constraint propagation to derive a compact representation of contact constraints in orbit space. By definition, a contact constraint $(p, \omega)$ is satisfied iff one of the shape constraints $\{((p, t), \omega): t \in T\}$ is satisfied. From (3) each shape constraint is satisfied if and only if its propagation to $t=0$ is satisfied. Consequently, a contact constraint is fully represented in orbit space $\mathcal{O}$ by a set of propagated constraints. We call that set the constraint locus and formally define it as:

Definition 7 (Constraint Locus): Let $(p, \omega)$ be a contact constraint, $\omega_{t}=P_{(p, t) \rightarrow\left(p_{t}, 0\right)}$ the propagation of $((p, t), \omega)$ to $t=0$ through the motion orbit $\Phi_{(p, t)}$, and $\left(p_{t}, 0\right)$ the corresponding point where the constraint gets propagated. The constraint locus of $(p, \omega))$ is the set $\mathcal{L}_{(p, \omega)}=$ $\left\{\left(\left(p_{t}, 0\right), \omega_{t}\right): t \in T\right\}$

To impose a contact constraint, we just need to construct its locus and chose an effector shape that at $t=0$ crosses the locus compliantly. Figure 8 shows examples of constraint locus for a rotational joint, a Hoekens linkage, and an elliptic trammel.

As illustrated in Figure 8, the constraint locus of a contact constraint is different, in general, from the orbits used to propagate it $t=0$. The following proposition gives an interesting relationship between orbits and loci: 

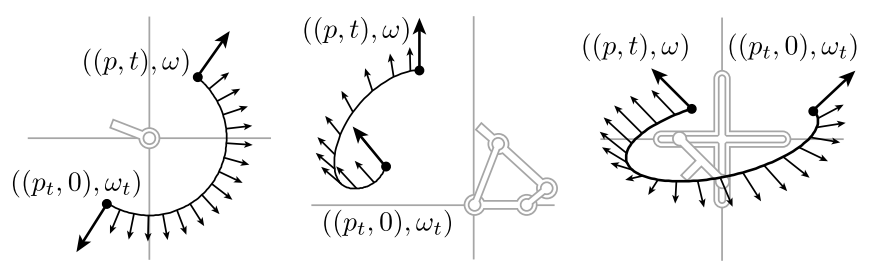

(a)
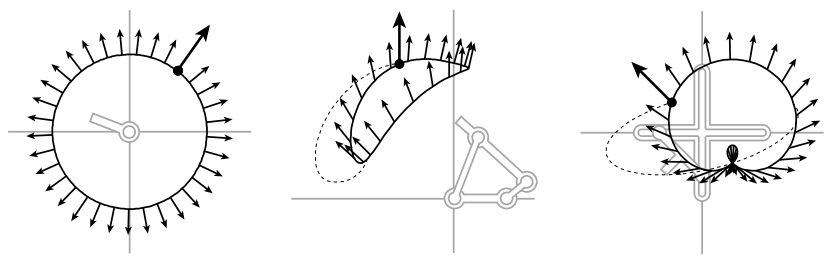

(b)

Fig. 8. Construction of constraint locus for three mechanisms: (left) rotational joint, (center) Hoekens linkage, and (right) elliptic trammel. (a) The constraint $((p, t), \omega)$ is propagated to $\left(\left(p_{t}, 0\right), \omega_{t}\right)$ at slice $t=0$, through a motion orbit. (b) The constraint locus is generated by repeating the process for all possible values of $t$.

Proposition 1 (Orbit vs. Locus): The locus of a contact constraint $(p, \omega)$ in orbit space is equal to the orbit of the

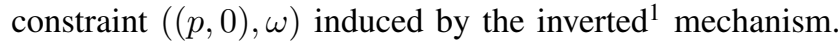

Proof: Let $A: T \longrightarrow \mathbb{S E}(2)$ be the mechanism map for the effector. We represent $A(t)$ as an homogenous matrix so that point $(p, 0)$ is mapped to $(A(t) \cdot p, t)$. The orbit associated with a constraint is the set $\Phi=\{(A(t) \cdot p, t): t \in T\}$ and its locus $\mathcal{L}=\left\{\left(q, t_{q}\right): A\left(t_{q}\right) \cdot q=p\right\}$. Then, for every point $q \in \mathcal{L}$, we have:

$$
A\left(t_{q}\right) \cdot q=p \Leftrightarrow q=A^{-1}\left(t_{q}\right) \cdot p
$$

that is, $q$ is in the locus induced by $A$ if and only if $q$ is in the orbit induced by $A^{-1}$, the inverted mechanism.

\section{Shape For Contact Problem}

We have now all the machinery in place to formulate the Shape for Contact problem. We first recall the key concepts:

- An effector is a map $E(s, t): \mathcal{S} \times T \mapsto \mathcal{W}$ describing the motion (parametrized by $t$ ) of a rigid curve (parametrized by $s$ ) driven by a mechanism.

- The shape of the effector is the curve $E(s, 0)$, describing the contact surface of the effector for configuration $t=0$ of the mechanism.

- The motion of the effector is captured by a vector field $\mathcal{M}(p, t)$ in $\mathcal{W} \times T$ that determines the direction of motion to follow by a particle at $p$ rigidly attached to the mechanism in configuration $t$.

- Motion orbits are the integral curves of $\mathcal{M}$, the trajectories followed by particles of the effector as actuated by the mechanism. They never intersect each other in the extended space $\mathcal{W} \times T$.

- The orbit space $\mathcal{O}$ of the effector is a one-to-one representation of the set of motion orbits. Our choice

\footnotetext{
${ }^{1}$ The inverse of a mechanism is obtained by exchanging moving and fixed reference frames.
}

of orbit space is the slice $t=0$ of $\mathcal{W} \times T$. The shape of the effector can be described as a curve $\alpha$ in $\mathcal{O}$.

- A shape constraint $((p, t), \omega)$ is an imposition on the effector to comply with $\omega$ at location $p$ and configuration $t$. It propagates along a motion orbit while held invariant in the moving frame. It is satisfied iff its propagation to $\mathcal{O}$ is satisfied, and it is represented in $\mathcal{O}$ as a point.

- A contact constraint $(p, \omega)$ is an imposition on the effector to locally comply with $\omega$ at location $p$ of the workspace for an unspecified configuration of the mechanism. It is satisfied iff any of the shape constraints in $\{((p, t), \omega)\}_{t \in T}$ is satisfied, and it is represented in $\mathcal{O}$ as a locus of constraints $\mathcal{L}_{(p, \omega)}$.

- An effector $E(s, t)$ locally satisfies a constraint $(p, \omega)$ if and only if its shape, described as a curve in $\mathcal{O}$, crosses the locus $L_{(p, \omega)}$ in compliance with the constraint.

The Shape for Contact problem formulates as:

Problem 1 (Shape for Contact): Let $\mathcal{M}$ be the motion field of an effector, $\left\{\left(p_{i}, \omega_{i}\right)\right\}_{i=1 \ldots N}$ a set of contact constraints, and $\mathcal{L}_{\left(p_{i}, \omega_{i}\right)}$ the corresponding loci in $\mathcal{O}$. Find a curve $\alpha$ in $\mathcal{O}$ that crosses all loci in compliance with the constraints.

Note that the loci described by different contact constraints may intersect each other. The points of the orbit space $\mathcal{O}$ where they intersect will likely induce inconsistent constraints if the $\alpha$ crosses them. To find a complete solution, we must find a shape that crosses all loci compliantly, without inconsistencies. If there are no inconsistent constraints, by construction the effector induced by the shape $\alpha$ locally satisfies all constraints.

The approach to propagate constraints allows us to express them in the reference pose of the effector at $t=0$, even without knowing at which pose $t$ each constraint will be enforced. The key is the use of the extended space $\mathcal{W} \times T$.

\section{EXAMPLE APPLICATION}

In this section we apply the Shape for Contact formulation to the problem of shaping the fingers of a two-fingered planar gripper to improve the stability of a grasp of a disk.

We consider the energetic model of grasp stability for a compliant simple gripper described in Mason et al. [15], similar to Hanafusa and Asada [39]. Every suitable handpose/object-pose configuration induces some level of grasp energy, supplied by motors and stored in springs. Assuming some dissipative forces, stable configurations of hand/object correspond to minima in that potential energy distribution. The shape of the potential energy in a neighborhood of a stable pose determines how stable it is. Sharp, narrow wells are less susceptible to be degraded by noise than broad shallow wells, and hence represent more stable grasps.

As illustrated in Figure 9, we model the actuation of a two-fingered planar gripper as a constant torque source $\tau_{m}$ compliantly coupled with springs to both fingers, providing a potential energy $U_{m}=\tau_{m} \cdot t$, where $t$ is the motor position or actuation parameter. The rest position of the fingers when the actuator is at $t$ is $\theta(t)$. Under compressing forces, each finger provides a potential $U_{i}=k_{f}\left(\theta_{i}-\theta(t)\right)^{2} / 2$, where $k_{f}$ 


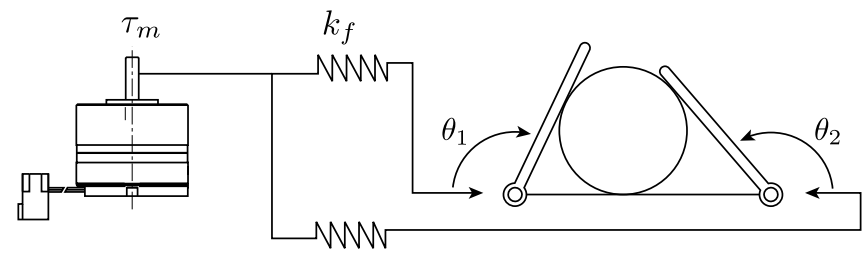

Fig. 9. The diagram illustrates the actuation/compliance scheme used to model hand/object interaction. Units are dimensionless throughout the analysis so that the diameter of the disk is 1 , the radius of the palm is 1 , the constant of finger springs is $k_{f}=1$, and when closing the hand, the motor is driven to a stall torque $\tau_{m}=1$.

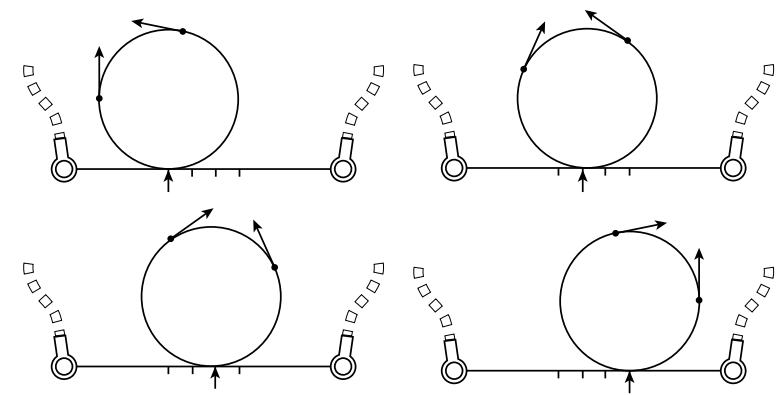

(a)

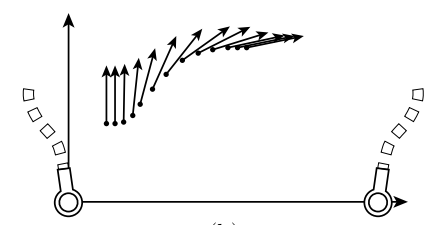

(b)

Fig. 10. (a) We chose contact points with the disk so that the left finger can easily push the disk only when it is to the left of the central stable pose. Opposite for the right finger. (b) Denser collection of the contact constraints imposed to the shape of the left finger. That collection is the input to the Shape for Contact problem.

is the spring constant of the finger and $\theta_{i}$ is the finger angle. The total energy of a grasp is then:

$$
U=U_{m}+\sum_{i=1}^{2} U_{i}=\tau_{m} \cdot t+\sum_{i=1}^{2} \frac{1}{2} k_{f}\left(\theta_{i}-\theta(t)\right)^{2}
$$

The shape of the potential energy $U$ depends on the finger-object contact geometry. By choosing different contact locations for different object poses, we can change the shape of that potential energy and improve the stability. Figure 10a shows a few selected contact points for different locations of a disk, each contact point constituting a contact constraint to satisfy. The problem of choosing contact constraints is important, but it is not the focus of this paper. For the rest of this section, we assume that we are given the set of desired constraints in Figure 10b.

As per Proposition 1 we transform the set of contact constraints into their corresponding loci in the orbit space. Figure 11a shows the loci for a finger actuated by a rotational joint and a finger actuated through a Hoekens linkage. To find a solution, we approximate the set of constraints by a vector field and integrate it numerically to find an integral

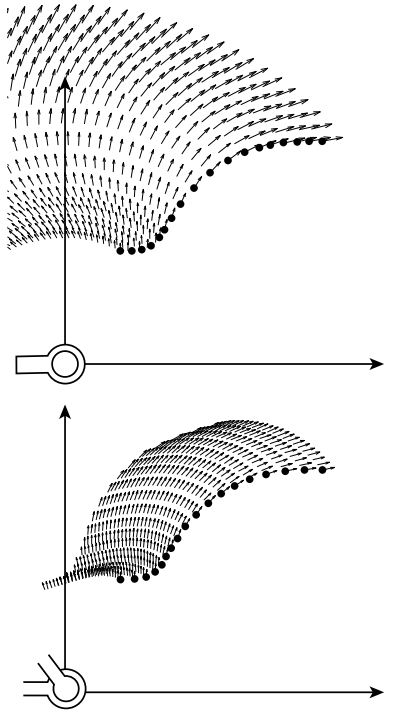

(a)

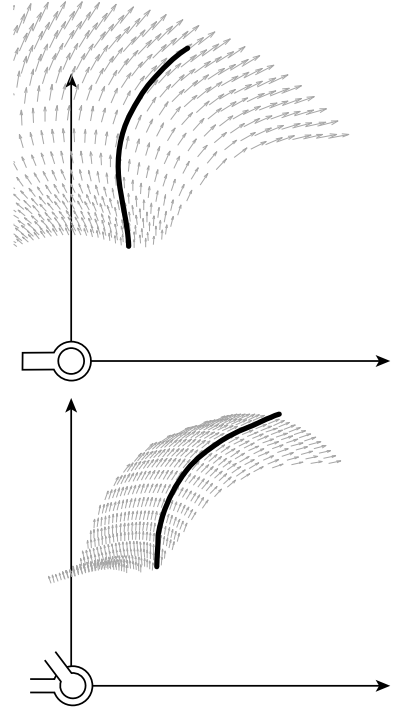

(b)

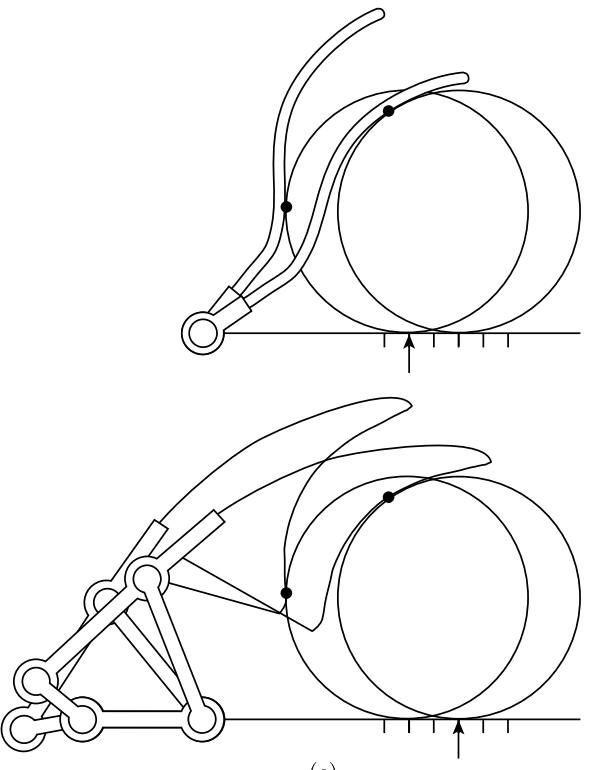

(c)

Fig. 11. Solution to the Shape for Contact problem for a (top) rotational joint and (bottom) Hoekens linkage. (a) Loci of all contact constraints (b) Integral curve of the approximated vector field. (c) Resulting effector.

curve (Figure 11b). The obtained curve results in an effector (Figure 11c) that complies with the imposed constraints and sharpens the potential energy wells of the central equilibrium grasp relative to straight fingers (Figure 12).

\section{DISCUSSION}

In this paper we study the role of effector shape in producing mechanical intelligence. Shape is a cheap design freedom relevant to manipulation, since it partly determines contact location. Shape is also unavoidable. Design choices have consequences, and in the design of an effector, we are always forced to chose a shape.

This paper assumes an actuation model for a mechanism, and a desired mechanical behavior expressed as a set of contact constraints, and provides tools to integrate that set 


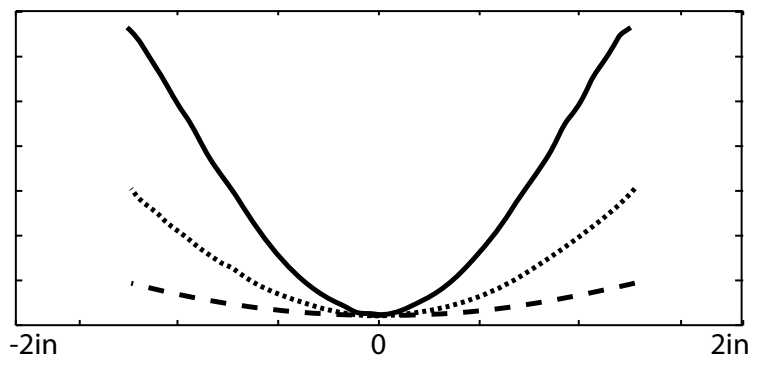

Fig. 12. Comparison between the stability of grasps induced by fingers designed for a Hoekens linkage (solid line), designed for a rotational joint (dotted line), and straight fingers with a rotational joint (dashed line). To enable the comparison, in each case we have zeroed the energy of the grasp configuration with minimal energy.

into an effector shape. For simplicity of exposition, we only consider tangential constraints. However, higher order constraints can be dealt with in a very similar manner. To guarantee that a desired contact is feasible, both the tangent and curvature of object and effector must be considered.

The proposed framework generalizes earlier work [1], focused on effectors actuated by revolute joints. In this paper we develop a more general formulation that covers all smooth 1 DOF planar mechanisms. We apply it to design fingers to improve the stability of a grasp, but the provided characterizations of shape and motion apply to all mechanisms in general.

A strength of the formulation is that it suggests further generalizations, that we plan to address in future work. First to spatial mechanisms, where contact vectors become contact planes, and integral curves become integral surfaces. Second to effectors with multiple degrees of freedom, where the motion field becomes a tensor field.

\section{REFERENCES}

[1] A. Rodriguez and M. T. Mason, "Grasp Invariance," The International Journal of Robotics Research, vol. 31, no. 2, pp. 237-249, 2012.

[2] N. T. Ulrich, "Grasping with Mechanical Intelligence," Master Thesis, University of Pennsylvania, 1989.

[3] J. F. Canny and K. Y. Goldberg, "RISC for Industrial Robotics: Recent Results and Open Problems," in IEEE International Conference on Robotics and Automation (ICRA), 1994, pp. 1951-1958.

[4] R. Pfeifer and F. Iida, "Morphological Computation: Connecting Body, Brain and Environment," Japanese Scientific Monthly, vol. 58, no. 2, pp. 48-54, 2005 .

[5] C. M. Gosselin, "Adaptive Robotic Mechanical Systems: A Design Paradigm," Journal of Mechanical Design, vol. 128, no. 1, pp. 192198, 2006

[6] M. H. Raibert, Legged Robots That Balance. The MIT Press, 1986.

[7] T. McGeer, "Passive Dynamic Walking," The International Journal of Robotics Research, vol. 9, no. 2, pp. 62-82, 1990.

[8] S. Collins, A. Ruina, R. Tedrake, and M. Wisse, "Efficient Bipedal Robots Based on Passive-Dynamic Walkers." Science, vol. 307, no. 5712, pp. 1082-1085, 2005.

[9] M. Gomes and A. Ruina, "Walking Model With no Energy Cost," Physical Review E, vol. 83, no. 3, pp. 6-9, 2011

[10] E. Lucas, "Huitieme Recreation-La Machine a Marcher," Recreat. Math, vol. 4, pp. 198-204, 1894.

[11] S. Hirose and Y. Umetani, "Soft Gripper," in International Symposium on Industrial Robots (ISIR), 1983, pp. 112-127.

[12] A. M. Dollar and R. D. Howe, "Towards Grasping in Unstructured Environments: Grasper Compliance and Configuration Optimization," Advanced Robotics, vol. 19, no. 5, pp. 523-543, 2005.
[13] L. Birglen, C. Gosselin, and T. Laliberte, Underactuated Robotic Hands. Springer, 2008.

[14] A. Rodriguez, M. T. Mason, and S. S. Srinivasa, "Manipulation Capabilities with Simple Hands," in International Symposium on Experimental Robotics (ISER), 2010.

[15] M. T. Mason, A. Rodriguez, S. S. Srinivasa, and A. S. Vazquez, "Autonomous Manipulation with a General-Purpose Simple Hand,' The International Journal of Robotics Research, vol. 31, no. 5, pp. 688-703, 2012.

[16] E. Z. Moore, D. Campbell, F. Grimminger, and M. Buehler, "Reliable Stair Climbing in the Simple Hexapod "RHex"," in IEEE Int. Conference on Robotics and Automation (ICRA), 2002, pp. 2222-2227.

[17] U. Saranli, M. Buehler, and D. E. Koditschek, "RHex: A Simple and Highly Mobile Hexapod Robot," The International Journal of Robotics Research, vol. 20, no. 7, pp. 616-631, 2001.

[18] R. Jardine, "Climbing Aids - US Patent 4184657," 1980.

[19] A. M. Dollar and R. D. Howe, "Joint Coupling Design of Underactuated Grippers," in Mechanisms and Robotics Conference (MR), 2006 , pp. 903-911.

[20] D. A. Theobald, W. J. Hong, A. Madhani, B. Hoffman, G. Niemeyer, L. Cadapan, J. J. Slotine, and J. K. Salisbury, "Autonomous Rock Acquisition," in AIAA Forum on Advanced Developments in Space Robotics, 1996.

[21] G. A. Kragten, M. Baril, C. M. Gosselin, and J. L. Herder, "Stable Precision Grasps by Underactuated Grippers," IEEE Transactions on Robotics, vol. 27, no. 6, pp. 1056-1066, 2011.

[22] G. Boothroyd and P. Dewhurst, Design for Assembly: A Designer's Handbook. Department of Mechanical Engineering, University of Massachusetts, Amherst, 1983.

[23] R. P. Berretty, K. Y. Goldberg, M. H. Overmars, and A. F. van der Stappen, "Trap Design for Vibratory Bowl Feeders," The International Journal of Robotics Research, vol. 20, no. 11, pp. 891-908, 2001.

[24] M. Peshkin and A. Sanderson, "Planning Robotic Manipulation Strategies for Workpieces that Slide," IEEE Journal on Robotics and Automation, vol. 4, no. 5, pp. 524-531, 1988.

[25] J. Wiegley, K. Y. Goldberg, M. Peshkin, and M. Brokowski, "A Complete Algorithm for Designing Passive Fences to Orient Parts," Assembly Automation, vol. 17, no. 2, pp. 129-136, 1997.

[26] M. Brokowski, M. Peshkin, and K. Y. Goldberg, "Optimal Curved Fences for Part Alignment on a Belt," Journal of Mechanical Design, vol. 117, no. 1, pp. 27-35, 1995

[27] M. T. Zhang and K. Y. Goldberg, "Gripper Point Contacts for Part Alignment," IEEE Transactions on Robotics and Automation, vol. 18 no. 6, pp. 902-910, 2002.

[28] D. E. Whitney, R. E. Gustavson, and M. P. Hennessey, "Designing Chamfers," The International Journal of Robotics Research, vol. 2, no. 4, pp. 3-18, 1983.

[29] F. Reuleaux, The Kinematics of Machinery: Outlines of a Theory of Machines. Macmillan, 1876.

[30] L. Joskowicz, "Reasoning about Shape and Kinematic Function in Mechanical Devices," Ph.D. dissertation, New York University, 1988.

[31] L. Joskowicz and S. Addanki, "From Kinematics to Shape: An Approach to Innovative Design," in National Conference on Artificial Intelligence (NCAI), 1988, pp. 347-352.

[32] B. Faltings, "Qualitative Kinematics in Mechanisms," Artificial Intelligence, vol. 44, no. 1-2, pp. 89-119, 1990

[33] K. D. Forbus, P. Nielsen, and B. Faltings, "Qualitative Spatial Reasoning: The CLOCK Project," Artificial Intelligence, vol. 51, no. 1-3, pp. 417-471, 1991.

[34] R. Gupta and M. J. Jakiela, "Simulation and Shape Synthesis of Kinematic Pairs via Small-Scale Interference Detection," Research in Engineering Design, vol. 6, no. 2, pp. 103-123, 1994.

[35] M. E. Caine, "The Design of Shape from Motion Constraints," Ph.D. dissertation, Massachusetts Institute of Technology, 1993.

[36] _ - "The Design of Shape Interactions Using Motion Constraints," in IEEE International Conference on Robotics and Automation (ICRA), 1994, pp. 366-371.

[37] C. Cai and B. Roth, "On the Spatial Motion of Rigid Bodies with Point Contact," in IEEE International Conference on Robotics and Automation (ICRA), 1987, pp. 686-695.

[38] D. J. Montana, "The Kinematics of Contact and Grasp," The International Journal of Robotics Research, vol. 7, no. 3, pp. 17-32, 1988.

[39] H. Hanafusa and H. Asada, "Stable Prehension by a Robot Hand with Elastic Fingers," in International Symposium of Industrial Robotics (ISIR), 1977, pp. 361-368. 